\title{
AVALIAÇÃO DA CITOTOXICIDADE E DA CAPACIDADE DE IMUNOMODULAÇÃO DE IL-1ß DE NOVOS DERIVADOS TIAZOLÍDINICOS EM PBMCS DE VOLUNTÁRIOS SADIOS E PACIENTES GOTOSOS
}

\author{
T. U. L. LINS ${ }_{2}^{1}$ J. F. BRANCO-JÚNIOR ${ }^{2}$, M. A. B. CORREIA ${ }^{1}$, K. M. VILAR ${ }^{1}$, V. A. S. \\ SILVA $^{1}$, N. G. CAVALCANTI ${ }^{3}$, C. D. L. MARQUES ${ }^{3}$, A. L. B. P. DUARTE ${ }^{3}$, M. J. B. M. \\ REGO$^{1}$, I. R. PITTA ${ }^{2}$, M. R. GALDINO-PITTA ${ }^{2}$, M. G. R. PITTA ${ }^{1}$ \\ ${ }^{1}$ Universidade Federal de Pernambuco, Núcleo de Pesquisa em Inovação Terapêutica Suely \\ Galdino, \\ Laboratório de Imunomodulação e Novas Abordagens Terapêuticas \\ ${ }^{2}$ Universidade Federal de Pernambuco, Núcleo de Pesquisa em Inovação Terapêutica Suely \\ Galdino, Laboratório de Síntese e Planejamento de Fármacos \\ ${ }^{3}$ Universidade Federal de Pernambuco, Hospital das Clínicas, Serviço de Reumatologia \\ E-mail para contato: thiago.ubiratan.lins@gmail.com
}

\begin{abstract}
RESUMO - A gota é uma doença onde a hiperuricemia sustentada leva a deposição de cristais de monourato de sódio (MSU) nos tecidos articulares seguido de um processo inflamatório local desencadeando ataques agudos tratados com anti-inflamatórios. Por sua vez, o Receptor Ativado por Proliferador de Peroxissomos- $\gamma$ (PPAR- $\gamma$ ) está relacionado com o controle do processo inflamatório nesses pacientes, atuando como modulador negativo, contribuindo para o desenvolvimento de agonistas do PPAR- $\gamma$, como as tiazolidinas. Dentre

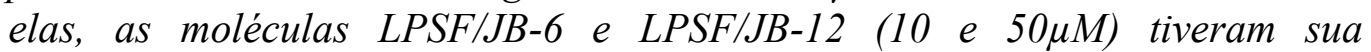
citotoxicidade avaliada em células mononucleares de voluntários sadios (PBMCs) pelo ensaio de MTT não sendo consideradas tóxicas. Foi realizado cultura de PBMCs de pacientes gotosos e voluntários sadios por $24 \mathrm{~h}$ à $37^{\circ} \mathrm{Ce} 5 \%$ de CO2 na presença dos LPSF/JBs $(25,50$ e $75 \mu \mathrm{M})$ a fim de, medir a secreção da citocina $I L-1 \beta(\mathrm{pg} / \mathrm{mL})$ pelo ensaio de ELISA. As condições tratadas foram comparadas ao estímulo com monourato de sódio. Nas células dos voluntários, a citocina foi inibida de maneira satisfatória pelo JB-6 25 (37,60 $\pm 134,9) ; 50$

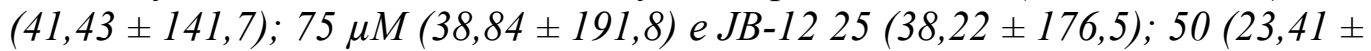
228,7); $75 \mu M(24,77 \pm 157,2$ e nas células dos pacientes pelo $J B-625(217,70 \pm$

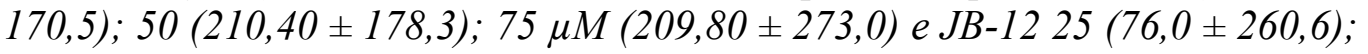
$50(69,14 \pm 174,0) ; 75 \mu M(54,43 \pm 253,0)$ sendo considerados candidatos a antiinflamatórios promissores para o tratamento das crises agudas de gota.
\end{abstract}

PALAVRAS-CHAVE: Tiazolidinas; Gota; MTT, atividade anti-inflamatória, Citocinas

ABSTRACT - Gout is a disease where sustained hyperuricemia leads to monourate sodium crystals (MSU deposition in the joint tissues followed by a local inflammatory process triggering acute attacks treated with anti- 
inflammatories. In turn, the Peroxisome Proliferator-Activated Receptor (PPAR$\gamma)$ is related to the inflammatory process control in these patients, acting as a negative modulator, contributing to PPAR- $\gamma$ agonists development such as thiazolidines. Among them, LPSF/JB-6 and $12(10$ and $50 \mu M)$ molecules had their cytotoxicity evaluated in mononuclear cells (PBMCs) of healthy volunteers by the MTT assay not being considered toxic. Gout patients and healthy volunteers $P B M C s$ were cultured for $24 \mathrm{~h}$ at $37^{\circ} \mathrm{C}$ and $\mathrm{CO}_{2} 5 \%$ in the presence of LPSF/JBS $(25,50$ and $75 \mu \mathrm{M})$ in order to measure the cytokine $I L-1 \beta$ secretion $(\mathrm{pg} / \mathrm{mL})$ by ELISA assay. The treated conditions were compared to sodium monourate stimulation. The cytokine was satisfactorily inhibited in volunteers cells by JB-6 25 (37.60 \pm 134.9$) ; 50(41.43 \pm 141.7) ; 75 \mu M(38.84 \pm 191.8)$ and $J B-1225$ (38.22 \pm 176.5$) ; 50(23.41 \pm 228.7) ; 75 \mu M(24.77 \pm 157.2)$ ands in patients cells by JB-6 25 (217.70 \pm 170.5$) ; 50$ (210.40 \pm 178.3$) ; 75 \mu M(209.80 \pm 273.0)$ and $J B-1225$ (76.0 \pm 260.6); 50 (69.14 \pm 174.0); $75 \mu M(54.43 \pm 253.0)$ being considered promising anti-inflammatory drugs candidates for the treatment of gout acute attacks.

KEYWORDS: Thiazolidines; Gout; MTT, anti-inflammatory activity, Cytokines

\section{INTRODUÇÃO}

A gota é uma doença inflamatória sistêmica ocasionada pela deposição de cristais de Monourato de Sódio nos tecidos articular e periarticular, sendo reflexo de períodos prolongados de hiperuricemia (AUS $\geq 6,8 \mathrm{mg} / \mathrm{dL}$ ), desencadeando um processo inflamatório agudo nas articulações devido ao englobamento dos cristais por células fagocíticas, proporcionando edema local, rubor e dor intensa. (SANTOS, 2016). Alguns estudos vêm mostrando que as crises agudas de gota são auto-limitadas, tendendo a chegar ao fim entre 710 dias, estando relacionado ao aumento da expressão do mRNA do Receptor Ativado por Proliferador de Peroxissomos Gama (PPAR- $\gamma$ ) (AKAHOSHI et al., 2003; CHANG et al., 2016). Desta forma, o desenvolvimento de moléculas agonistas do receptor PPAR- $\gamma$ poderia minimizar os sintomas da gota atuando na diminuição dos níveis de citocinas próinflamatórias como IL-1 $\beta$, IL-6 e TNF- $\alpha$. Assim este trabalho visou testar novos derivados tiazolidínicos buscando atividade anti-inflamatória, avaliando a citotoxicidade em células saudáveis e a imunomodulação da citocina IL-1 $\beta$ em PBMCs de voluntários sadios e pacientes gotosos.

\section{MATERIAIS E MÉTODOS}

O projeto foi previamente aprovado pelo Comitê de Ética (CEP/CCS/UFPE), sob o registro 19646913.0.0000.5208. Foram coletados $27 \mathrm{~mL}$ de sangue em tubos de heparina de cada um dos 9 pacientes e 4 voluntários saudáveis. As Células Mononucleares de Sangue Periférico (PBMCs) foram centrifugadas em gradiente de Ficoll-Paque PLUS. Para o ensaio de citotoxicidade foram utilizadas PBMCs de três voluntários saudáveis em triplicata sendo plaqueadas em placas de 96 poços $\left(5 \times 10^{5}\right.$ células/poço) e incubadas em estufa a $37^{\circ} \mathrm{C}$ e $5 \%$ de $\mathrm{CO}_{2}$ por $48 \mathrm{~h}$ com os compostos LPSF/JB-6 e $12(10$ e $75 \mu \mathrm{M})$ tendo sua viabilidade avaliada através do ensaio de MTT. Paralelamente, foram preparados os cristais de monourato de sódio (MSU). As PBMCs coletadas foram cultivadas por $24 \mathrm{~h}$ em placas de 24 poços $\left(1 \times 10^{6}\right.$ cel $/ \mathrm{mL})$, com os cristais de MSU sintetizados $(0,5 \mathrm{mg} / \mathrm{mL})$, os fármacos de referência Colchicina $(2,5 \mu \mathrm{M})$ e Metilpredinisolona $(100 \mu \mathrm{M})$ e compostos LPSF/JB-6 e 12 (25, 50 e 
$75 \mu \mathrm{M})$. Após a cultura, o sobrenadante foi coletado e realizado ELISA para a dosagem da citocina IL-1 $\beta$ (BD Bioscience - limites de detecção 3,90-500 pg/mL) seguindo as instruções do fabricante. O software GraphPad Prism 6 foi utilizado para confecção dos gráficos e análise estatística (testes de Kolmogorov-Smirnov e Wilcoxon; valor de $\mathrm{p}>0,05$ como estatisticamente significativo).

\section{RESULTADOS E DISCUSSÃO}

\subsection{Ensaio de Citotoxicidade}

Nas doses testadas, os compostos não se apresentaram tóxicos frente às PBMCs, não interferindo na viabilidade celular (Tabela 1).

Tabela 1 - Viabilidade celular de PBMCs de voluntários sadios (n=3) após exposição aos derivados tiazolidínicos LPSF/JBs 6 e 12 nas doses de 10 e $75 \mu \mathrm{M}$

\begin{tabular}{lcccc}
\hline Compostos / Doses $(\mu)$ & \multicolumn{3}{c}{10} & \multicolumn{2}{c}{75} \\
\hline \multicolumn{4}{c}{ Viabilidade (\%)Desvio padrão (\%)Viabilidade (\%)Desvio padrão (\%) } \\
\hline LPSF/JB-6 & 93,62 & 11,04 & 95,56 & 7,7 \\
LPSF/JB-12 & 95,48 & 7,83 & 97,82 & 1,94 \\
\hline
\end{tabular}

\subsection{Dosagem de IL-1B por ELISA}

Os resultados estão expressos em valores de medianas com seus respectivos desviospadrão. As comparações foram feitas entre as condições tratadas com a condição do estímulo C + MSU. Para os voluntários sadios, o grupo C + MSU (41,64 $\pm 75,32 \mathrm{pg} / \mathrm{mL})$ mostrou diferença significativa comparado com o grupo não tratado $(3,9060 \pm 15,93 \mathrm{pg} / \mathrm{mL} ; \mathrm{p}=$ 0,1250) (Figura 1 - A e B). Destacaram-se a Metilprednisolona $(43,69 \pm 67,97 \mathrm{pg} / \mathrm{mL} ; \mathrm{p}=$ 0,6250), o LPSF/JB-6 nas doses de 25 (37,60 $\pm 134,9 \mathrm{pg} / \mathrm{mL} ; \mathrm{p}>0,99), 50(41,43 \pm 141,7$ $\mathrm{pg} / \mathrm{mL} ; \mathrm{p}=0,8750)$ e $75 \mu \mathrm{M}(38,84 \pm 191,8 \mathrm{pg} / \mathrm{mL} ; \mathrm{p}>0,99)$ e o LPSF/JB-12 nas doses de $25(38,22 \pm 176,5 \mathrm{pg} / \mathrm{mL} ; \mathrm{p}=0,8750), 50(23,41 \pm 228,7 \mathrm{pg} / \mathrm{mL} ; \mathrm{p}=0,8750)$ e $75 \mu \mathrm{M}(24,77$ $\pm 157,2 \mathrm{pg} / \mathrm{mL} ; \mathrm{p}=0,8750$ ). (Figura 1 - A e B).

Para os pacientes, o grupo C + MSU $(165,0 \pm 192,0 \mathrm{pg} / \mathrm{mL})$ mostrou diferença significativa comparado com o grupo não tratado $(3,91 \pm 2,01 \mathrm{pg} / \mathrm{mL} ; \mathrm{p}=0,0039)$ (Figura 1 $\mathrm{C}$ e D). Destacaram-se a Metilprednisolona $(87,82 \pm 51,80 \mathrm{pg} / \mathrm{mL} ; \mathrm{p}=0,0078)$, o LPSF/JB-6 nas doses de $25(217,70 \pm 170,5 \mathrm{pg} / \mathrm{mL} ; \mathrm{p}=0,91), 50(210,40 \pm 178,3 \mathrm{pg} / \mathrm{mL} ; \mathrm{p}=0,9102)$ e $75 \mu \mathrm{M}(209,80 \pm 273,0 \mathrm{pg} / \mathrm{mL} ; \mathrm{p}=0,9102)$ e o LPSF/JB-12 nas doses de $25(76,0 \pm 260,6$ $\mathrm{pg} / \mathrm{mL} ; \mathrm{p}=0,4258), 50(69,14 \pm 174,0 \mathrm{pg} / \mathrm{mL} ; \mathrm{p}=0,0742)$ e $75 \mu \mathrm{M}(54,43 \pm 253,0 \mathrm{pg} / \mathrm{mL} ; \mathrm{p}$ $=0,6523$ ). (Figura $1-\mathrm{C}$ e D). O LPSF/JB-12 se destacou quanto a sua atividade antiinflamatória. Assim foi possível foi possível demonstrar que derivados tiazolidínicos com substituintes mais volumosos podem ser bastante úteis no desenvolvimento de moléculas antiinflamatórias (BARROS et al., 2010; CHADHA et al., 2015). 
Figura 1 - Secreção de IL-1 $\beta$ produzida por PBMCs de voluntários saudáveis (A e B) e pacientes portadores de gota (C e D). Significância estatística MSU e demais grupos (* ou **); significância estatística entre MSU + Colchicina e demais grupos (\# ou \#\#).

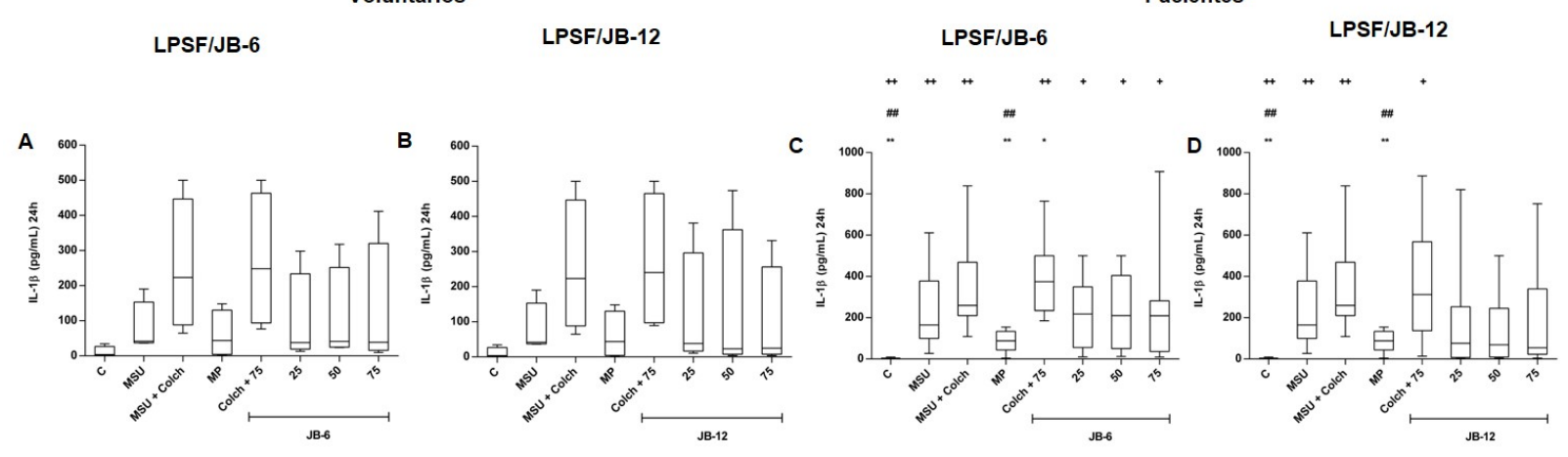

\section{CONCLUSÃO}

Os derivados tiazolidínicos podem ser considerados promissores principalmente aqueles substituídos na posição 3,5-dimetil por grupos volumosos ou básicos podendo ser uma boa alternativa para o tratamento das crises agudas, atuando como bons candidatos a fármacos anti-inflamatórios para o tratamento da gota, uma vez que inibe citocinas pró-inflamatórias importantes para fisiopatologia da doença. Contudo, ainda se faz necessário a dosagem de outras citocinas e proteínas envolvidas na formação do inflamossomo para entender melhor o seu mecanismo de ação, tais como: NLRP3, caspase 1, IL-6, IL-22, IL-18 e IL-10.

\section{REFERÊNCIAS}

AKAHOSHI, T. et al. Rapid induction of peroxisome proliferator-activated receptor $\gamma$ expression in human monocytes by monosodium urate monohydrate crystals. Arthritis \& Rheumatism, v. 48, n. 1, p. 231-239, jan. 2003.

BARROS, C. D. et al. Synthesis and anti-inflammatory activity of new arylidenethiazolidine-2,4-diones as PPARgamma ligands. Bioorg Med Chem, v. 18, n. 11, p. 3805-3811, 2010.

CHADHA, N. et al. Thiazolidine-2,4-dione derivatives: Programmed chemical weapons for key protein targets of various pathological conditions. Bioorganic \& Medicinal Chemistry, v. 23, n. 13, p. 2953-2974, 1 jul. 2015.

CHANG, W.-C. et al. Genetic variants of PPAR-gamma coactivator 1B augment NLRP3mediated inflammation in gouty arthritis. Rheumatology, v. 56, n. 3, p. kew337, 25 out. 2016.

SANTOS, F. Gota: uma revisão. Revista Médica da UFPR, v. 3, n. 1, p. 25-31, 2 maio 2016.

\section{AGRADECIMENTOS}

UFPE, FACEPE, CNPq, CAPES e INCT-IF 